\title{
On the stationary state of a network of inhibitory spiking neurons
}

\author{
Wolfgang Kinzel * \\ University of Würzburg \\ Institute of Theoretical Physics and Astrophysics \\ Am Hubland, Würzburg, Germany
}

November 13, 2018

\begin{abstract}
The background activity of a cortical neural network is modeled by a homogeneous integrate-and-fire network with unreliable inhibitory synapses. Numerical and analytical calculations show that the network relaxes into a stationary state of high attention. The majority of the neurons has a membrane potential just below the threshold; as a consequence the network can react immediately - on the time scale of synaptic transmission- on external pulses. The neurons fire with a low rate and with a broad distribution of interspike intervals. Firing events of the total network are correlated over short time periods. The firing rate increases linearly with external stimuli. In the limit of infinitely large networks, the synaptic noise decreases to zero. Nevertheless, the distribution of interspike intervals remains broad.
\end{abstract}

\section{Introduction}

A neural network processes information by cooperating neurons, which interact by exchanging spikes of action potentials. Numerous experimental and theoretical findings suggest that a cortical neural network has a background activity of neurons firing irregularly at low rates

*email: kinzel@physik.uni-wuerzburg.de 
[1, 8. On top of this noisy activity, information is processed. It is still not known how a neural network codes, stores and processes information. For instance, information in the cortex may be coded in patterns of neuronal firing rates (Hebbian cell assemblies) or in spatiotemporal patterns of spikes (synfire chains). But, evidently, all this occurs in a state of noisy activity. Therefore it is important to understand the properties of the background activity.

Quantitative estimates of the rate and size of the synaptic events in the cortex suggest that the irregular background activity stems from a balance between excitatory and inhibitory postsynaptic currents [13, 14. Both of these currents are large, but they compensate and the activity of the network is triggered by the fluctuations of the total current. Recent model calculations showed how to integrate Hebbian cell assemblies or synfire chains in a balanced network [3, 4].

However, experimental evidence for balanced synaptic input is still not available and it is not understood whether and how a neural network is able to compensate large synaptic currents of different directions [13, 17]. In addition, inhibitory pulses can shunt excitation very effectively. Therefore I follow a different approach and investigate a network with inhibitory synapses, only. At a first step, the investigations concentrate on one single mechanism: unreliable inhibition.

It is text book knowledge that synaptic transmission is a stochastic process. In fact, experiments on single synapses indicate that synapses transmit siganls with a probability which can be as low as a few percent [1, 2]. Only if an incoming spike is repeated in a short time interval, the transmission probability increases [12]. This indicates that the background activity is driven by unreliable synapses whereas for information processing teh network improves synaptic reliability.

In this paper I investigate how unreliable inhibition effects the collective properties of the network, hence I do not consider any kind of additional spatial or temporal noise, neither disorder in the synaptic connections and thresholds nor noise in the external stimulus.

One of the simplest models to describe the cooperative properties of interacting neurons is a network of integrate-and-fire (IF) units [8]. A single neuron is driven by an excitatory input and generates and transmits spikes. The total network may have unexpected complex properties which are not obvious from a single model neuron.

From a more general point of view, IF networks are networks of pulse coupled oscillators, and a large amount of work has been devoted to the investigation of the dynamics of nonlinear oscillators [15]. Several interesting phenomena have been found for IF-networks, for instance synchronization [10], phase locking, clustering [7], fast global oscillations [6], stochastic resonance, deterministic and transient chaos 
[16, 19] and

In this paper I investigate the stationary state of a network of inhibitory neurons. The effect of unreliable inhibitory synapses on the global properties of the network is studied numerically as well as analytically. A single synapse consists of stochastic components; it transmits signals with a probability which can be as low as a few percent [1, 2, 12]. Any other microscopic mechanisms are omitted in the model, like synaptic delay, distribution of thresholds and synaptic strengths. The effect of excitatory input is modeled by a constant stimulus.

Neither the model nor the analytic methods of this paper are new. Nevertheless, a new phenomenon is observed which, to my knowledge, was not discussed before: A network with inhibitory couplings relaxes to a stationary state of high attention. After a few spikes per neuron, the distribution of membrane potentials is sharply peaked just below threshold. As a consequence, the system is able to react immediately, i.e. on the time scale of spike generation, to tiny external pulses.

In the model of this paper the synaptic noise disappears in the limit of infinitely large networks. However, the system adjusts to a stationary state where the distribution of interspike intervals remains broad.

\section{The model}

In our model, each neuron is described by the following differential equation for the membrane potential $V(t)$ [8],

$$
\tau \frac{d V}{d t}=-V(t)+V_{\infty}
$$

$\tau$ is the relaxation time for the membrane potential and the driving potential $V_{\infty}$ models a constant excitatory stimulus. When the membrane potential $V(t)$ reaches the threshold value $\theta$ the neuron fires a spike and resets its potential to the value $V_{r}$.

A homogenous network is studied where each neuron has the parameters $\tau=10 \mathrm{~ms}, V_{r}=-70 \mathrm{mV}, V_{\infty}=-50 \mathrm{mV}$ and $\theta=-51 \mathrm{mV}$. Hence, without any interactions, each neuron would fire periodically with the time period $T=\tau \ln 20 \simeq 30 \mathrm{~ms}$ which corresponds to a frequency of $33 \mathrm{~Hz}$.

I consider a homogeneous network of $N$ mutually coupled neurons. If any neuron fires, it sends its spike to its synapses which are connected to all other neurons. The synapse is unreliable, it transmits the signal with a probability $p$, only. If a signal is transmitted, it reduces the postsynaptic potential by an amount $J$. In our simulations, I use 
the parameters $N=10000, p=0.5$ and $J=0.002 \mathrm{mV}$. Hence, each synapse is extremely weak; but the effective strength $p J N=10 \mathrm{mV}$ is of the order of the difference between threshold and reset potentials.

Between two consecutive pulses in the total the network, each neuron follows Eq. (11) which is easily solved. The time needed to increase the potential $V\left(t_{1}\right)$ to a value $V\left(t_{2}\right)$ is given by

$$
t_{2}-t_{1}=\tau \ln \frac{V_{\infty}-V\left(t_{1}\right)}{V_{\infty}-V\left(t_{2}\right)}
$$

This equation allows a simple numerical simulation of the total network without solving any differential equation. At each computational step, the algorithm searches for the neuron with the maximal values of $V(t)$, calculates the time this neuron needs to reach the threshold $\theta$ and resets its potential to the value $V_{r}$. Then the potentials of all other neurons are calculated from Eq.(2) and, with probability $p$, their values are reduced by the amount of $J$. This process is iterated until the system has reached a stationary state. Then the distributions of potentials and interspike intervals are recorded.

\section{Results of the simulations}

The homogeneous network of IF-neurons with the parameters given before has been simulated numerically. I start from a flat distribution of potentials between reset and threshold value. After a few spikes per neuron the network has lost the memory to its initial state and has relaxed to a stationary state. Note that our model is stochastic, hence I have to discuss distributions of quantities of interest.

In the following I show results for the distribution of membrane potentials and interspike intervals, both of which are accessible for real neurons. Fig 1 shows the membrane potential of one single neuron as a function of time. Since the network is homogeneous all neurons have identical properties. Between two spikes, each neuron receives about 5000 pulses from the 9999 other neurons of the network. Each pulse reduces its potential by the amount of $J=0.002 \mathrm{mV}$.

I see that a typical neuron increases its potential exponentially fast to a kind of plateau immediately below threshold. The stochastic input leads to fluctuations of the membrane potential. Whenever this potential exceeds the threshold value the neuron fires. Note that the average spike interval increases from $30 \mathrm{~ms}$ without couplings to about $100 \mathrm{~ms}$. Obviously, the inhibitory pulses reduce the firing rate. But less obvious, most of the neurons have a membrane potential close to the threshold value. This is shown in Fig. 2, The distribution of 


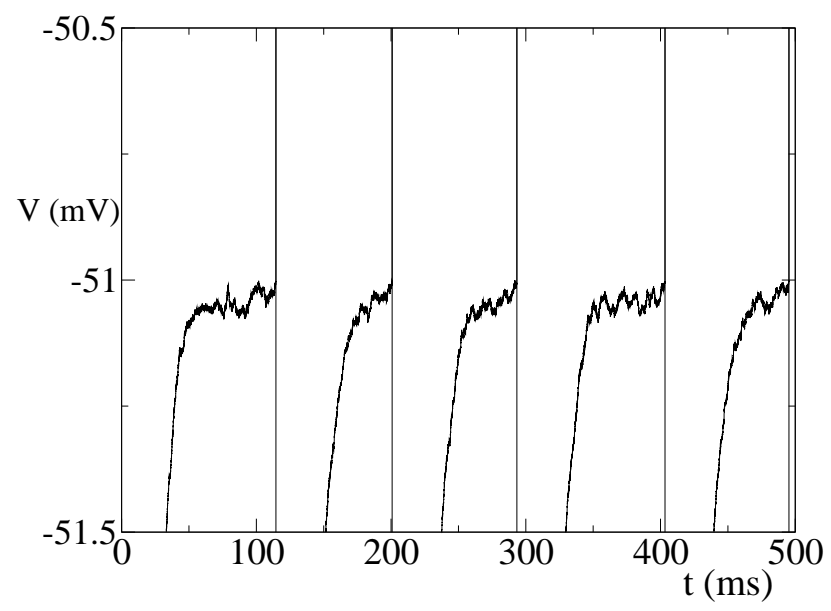

Figure 1: Membrane Potential $V(t)$ as a function of time for a network of 10000 inhibitory neurons with unreliable synapses. As soon as the potential reaches the threshold value $\theta=-51 \mathrm{mV}$ the neuron emits a spike and resets its potential to the value $V_{r}=-70 \mathrm{mV}$. Between two spikes, the potential is reduced about 5000 times by the value of $J=0.002 \mathrm{mV}$

membrane potentials has a peak which is only $0.05 \mathrm{mV}$ below threshold.

Note that the initial distribution was flat between $-70 \mathrm{mV}$ and -50 $\mathrm{mV}$. After a short time the system has relaxed to a stationary where most of the neurons have a potential immediately below threshold. As a consequence, a large fraction of neurons can react immediately to tiny pulses of the order of a fraction of millivolts. In Fig 3 the fraction of neurons is shown which fires immediately after a excitatory pulse of a given strength $J_{e x}$. For comparison, I have included the corresponding curve for the initial state. Fig. 4 shows the corresponding spike pattern for $J_{e x}=0.5 \mathrm{mV}$. All of the neurons which have responded to the pulse excitation remain quiet for at least $50 \mathrm{~ms}$, see Fig 5 . These results show that the network has relaxed to a state of high attention and can react to incoming small pulses on the time scale of synaptic transmission.

The distribution of interspike intervals $T$ is shown in Fig. 5. There are no intervals below $50 \mathrm{~ms}$ and the distribution decays exponentially fast for large values of $T$. Its mean value is $\langle T\rangle=97.6 \mathrm{~ms}$ and its standard deviation $\sigma=29.0 \mathrm{~ms}$, which gives a $C V$-value of 0.3 . Since the network has identical neurons, the average time $s$ between two consecutive spikes in the total network is $\langle s\rangle=\langle T\rangle / N \simeq 0.01$ ms. Usually one assumes that the spikes arriving at a single neuron 


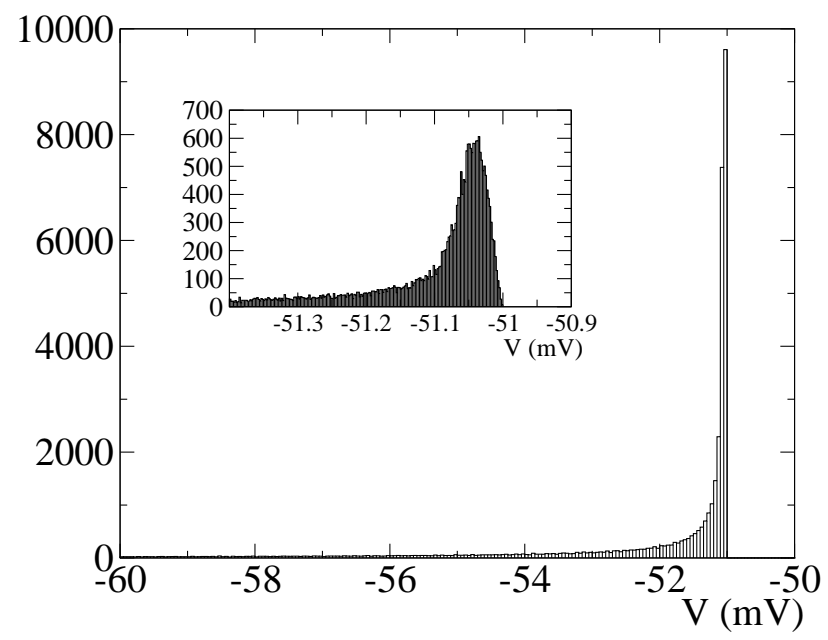

Figure 2: Histogram of the membrane potentials of the network at some specified time. Initially, the distribution was flat between $-70 \mathrm{mV}$ and -51 $\mathrm{mV}$. In the stationary state, shown here, most of the neurons have a potential immediately below threshold threshold. The inset shows the same distribution close to threshold.

are uncorrelated, which would yield a Poisson, i.e. an exponential distribution of the intervals $s$ between synaptic events. Fig. 6] shows that this is not true. Although small values of $s$ are more frequent than larger ones, the distribution is not Poissonian indicating correlations between events. In fact, the inset of Fig. [6 shows the correlations between consecutive spikes. The spike intervals of the total network are ordered, $\left(s_{1}, s_{2}, s_{3}, \ldots s_{j}, ..\right)$, and Fig. 6 shows the cross correlation

$$
C(k)=\frac{<s_{j+k} s_{j}>-<s_{j}>^{2}}{<s_{j}^{2}>-<s_{j}>^{2}}
$$

Correlations can also be observed from the statistics of spike counts. For instance, the Fano factor $F$ is defined as the ratio of the variance divided by the average number of spikes observed in some time interval $\Delta T$. For a Poisson process one has $F=1$ independent of $\Delta T$. I have recorded the number of network events for $\Delta T=1 \mathrm{~ms}$ and find a much smaller Fano factor of $F=0.15$. Hence the distribution of the number of spikes is much smaller than the corresponding one for uncorrelated synaptic inputs.

In the context of pulse coupled oscillators the phase $\phi$ of an oscillator is of interest. When a reference neuron fires at time $t$ the phase is defined as $\phi_{k}=t-t_{k}$ where neuron $k$ has fired at at previous time 


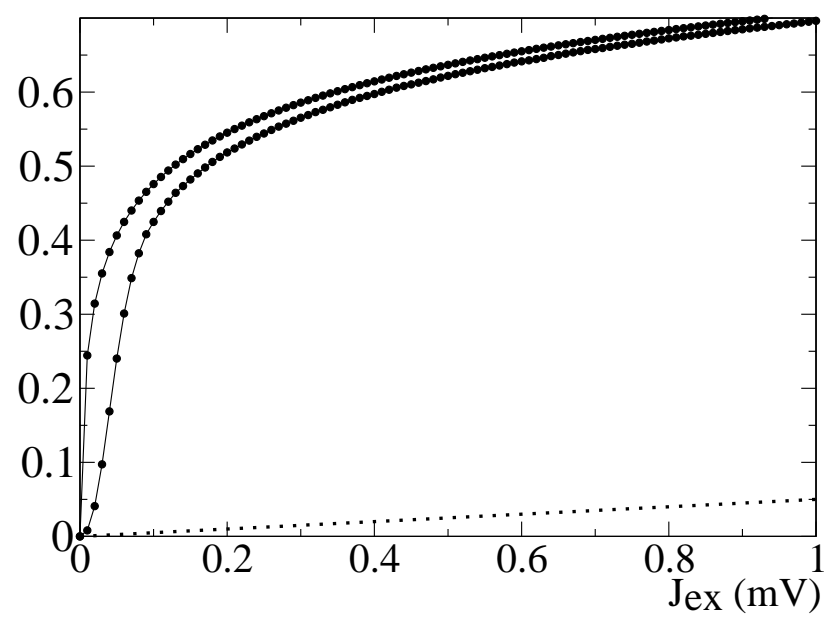

Figure 3: The fraction of neurons which fires immediately after an excitatory pulse of a given value $J_{e x}$. The network with stochastic synapses (lower values) is compared with a corresponding deterministic network (upper values). The dotted line shows the corresponding fraction of neurons for the initial state with a flat distribution of potentials.

$t_{k}$. The distribution of phases is shown in Fig. 7. The distribution of a deterministic network which is flat between 0 and $100 \mathrm{~ms}$ (see below) is rounded due to synaptic noise.

It may be interesting to compare the properties of our stochastic model with the corresponding deterministic one, i.e. with the model with parameters $p=1$ and $J=0.5$. The effective strength $p J N=10$ $\mathrm{mV}$ is identical but the synapses function completely reliable. Our simulations show that the deterministic model relaxes to a periodic state with a flat distribution of phases. Each neuron fires periodically with the period $T=100.04 \mathrm{~ms}$. The time $s$ between events of the total network is constant, $s=T / N=0.10004 \mathrm{~ms}$. Hence, both of the distributions of interspike as well as interevent intervals have a sharp peak at a single value. This property of the deterministic network is definitely at variance with the behavior of real neural networks.

Nevertheless, the distribution of membrane potentials is similar to the one of the corresponding stochastic network, Fig. 2. Only very close to threshold one observes deviations. Note that a flat distribution of phases transforms to a distribution of potentials which is proportional to $1 /(\mu-u)$ with a cutoff above the value $\theta$. The parameter $\mu$ is slightly larger than the value $\theta$ and will be calculated in the following section. 


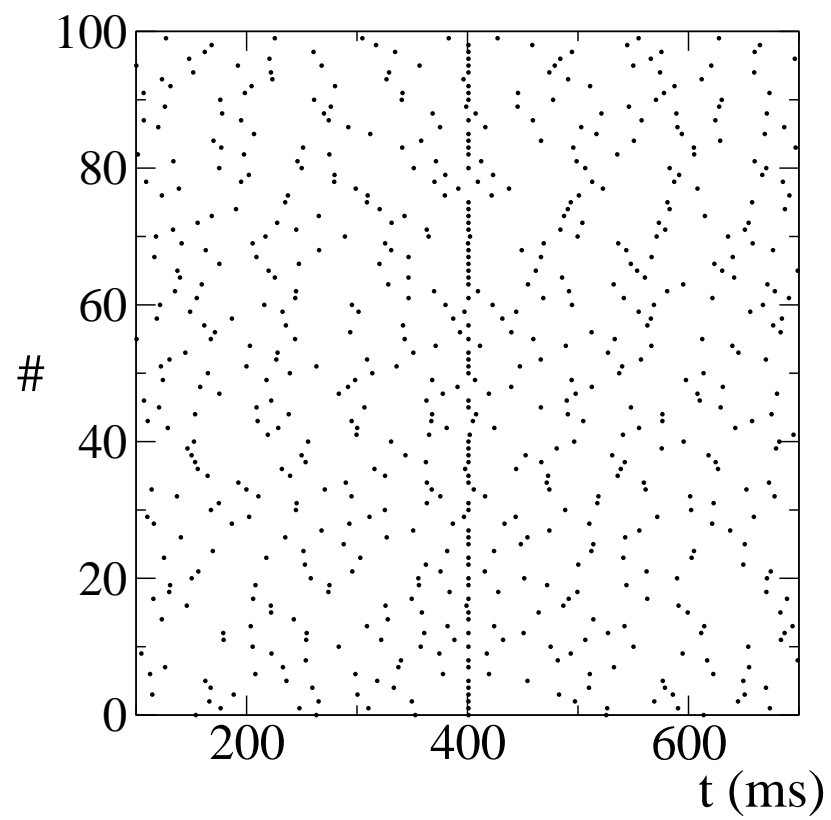

Figure 4: Spike patterns of one hundred neurons (out of 10000). At time $t=400 \mathrm{~ms}$, an excitatory pulse is applied which increases all postsynaptic potentials by the amount of $0.5 \mathrm{mV}$. According to the previous figure 3 , about $60 \%$ of the neurons fire immediately.

\section{Analytic approximations}

Although our model is rather simple it cannot be calculated analytically. Even two neurons with unreliable synapses yield a complex multifractal distribution of interspike intervals which can only be calculated numerically [11]. But analytic approximations are possible and help to understand the influence of the model parameters on the behavior of the network.

It is well known that a stochastic network may be approximated by a diffusion process of the membrane potential [18, 8, 5. Assuming uncorrelated synaptic input one can describe the time dependent distribution of potentials by a Fokker-Planck equation for an OrnsteinUhlenbeck process. Spike intervals correspond to first passage times. Although, even for this approximation, there is no closed expression for the distribution of spike intervals, one can calculate its mean value from the solution of an ordinary differential equation.

Two parameters enter this approximation: The mean value and the variance of the synaptic input. Let us first ignore the fluctuations and consider the membrane potential, Eq.(1), averaged over the stochastic 


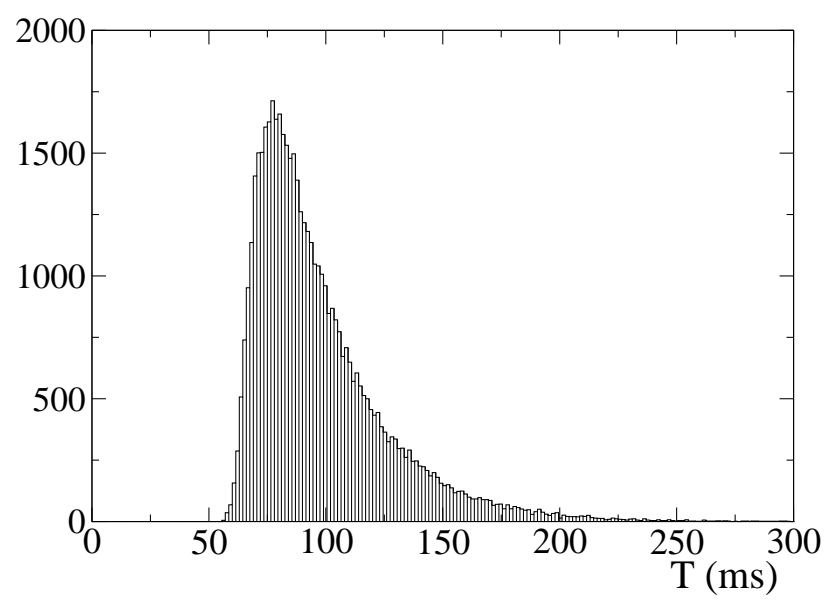

Figure 5: Histogram of the intervals between spikes of a single neuron.

synaptic input. Each spike in the network adds a pulse $p J$ to the potential of every neuron. The average time between two pulses is given by $\langle s\rangle=\langle T\rangle / N$, hence I obtain the following equation

$$
\tau \frac{d V}{d t}=-V(t)+V_{\infty}-\frac{\tau p J N}{<T>}
$$

The mean value of the membrane potential moves exponentially fast to the value

$$
\mu=V_{\infty}-\frac{\tau J g N}{<T>}
$$

until it crosses the threshold. If I would neglect fluctuations, the spike interval would follow from Eq.(2), which yields

$$
\frac{<T>}{\tau}=\ln \frac{V_{\infty}-\frac{\tau p J N}{<T>}-V_{r}}{V_{\infty}-\frac{\tau p J N}{<T>}-\theta}
$$

This self-consistent equation for the interspike interval is exact for the deterministic model in the limit of a large network, $N \rightarrow \infty$ with $J N=$ const. Note that in this case, the system relaxes to a periodic state with only one single spike interval $T$. If the effective synaptic strength $J N$ is large, i.e. if the spike interval $T$ is much large than $\tau$, the denominator of Eq.(6) approaches zero and one obtains

$$
\frac{<T>}{\tau}=\frac{p J N}{V_{\infty}-\theta}
$$

For a given value of $J$, the spike interval is proportional to the size of the network and its inverse, the spike rate, is proportional to the difference between stimulus and threshold potential. 


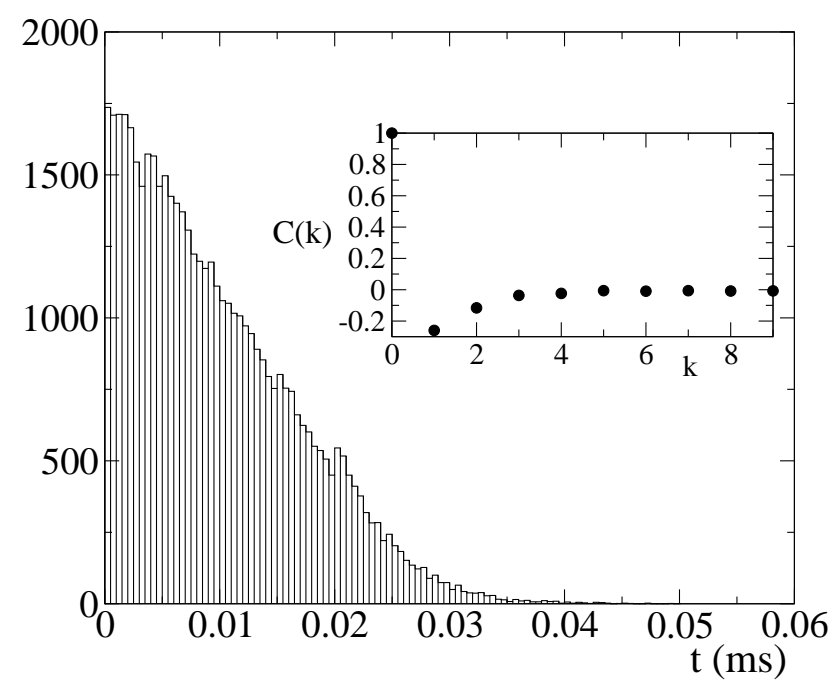

Figure 6: Histogram of the intervals between spikes of the total network. The inset shows the corresponding cross correlation of consecutive spikes.

Our numerical simulations showed that the deterministic network relaxes to a state with a flat distribution of phases (times after firing). Using this fact, one immediately derives the distribution of membrane potentials from Eq.(11) with the result

$$
p(V)=\frac{\tau N}{T} \frac{1}{\mu-V} \quad \text { for } \quad V_{r}<V<\theta
$$

In the limit of infinitely large networks the potential $\mu$ approaches the threshold, hence in this limit almost all neurons have a potential very close to threshold. In fact, Eqs. (617) give the fraction $P(\varepsilon)$ of neurons which have a membrane potential in the interval $[\theta-\varepsilon, \theta]$,

$$
P(\varepsilon)=\frac{\ln (\mu-\theta+\varepsilon)-\ln (\mu-\theta)}{\ln \left(\mu-V_{r}\right)-\ln (\mu-\theta)}
$$

This fraction approaches $P(\varepsilon)=1$ with $\mu \rightarrow \theta$.

For the stochastic version of the model, the simulations of the previous section yielded a broad distribution of spike intervals $T$ which is caused by the fluctuations of synaptic inputs. Therefore, in the analytic approximation, fluctuations of the synaptic input are included by adding noise to Eq. (11) with mean value $\mu$ and variance $\sigma^{2}$. The value of the mean $\mu$ is given by Eq.(5) and the variance can be estimated by assuming a Poisson statistics, one finds [5]

$$
\mu=V_{\infty}-\frac{\tau J g N}{<T>}, \quad \sigma^{2}=J^{2} \frac{p N \tau}{<T>}
$$




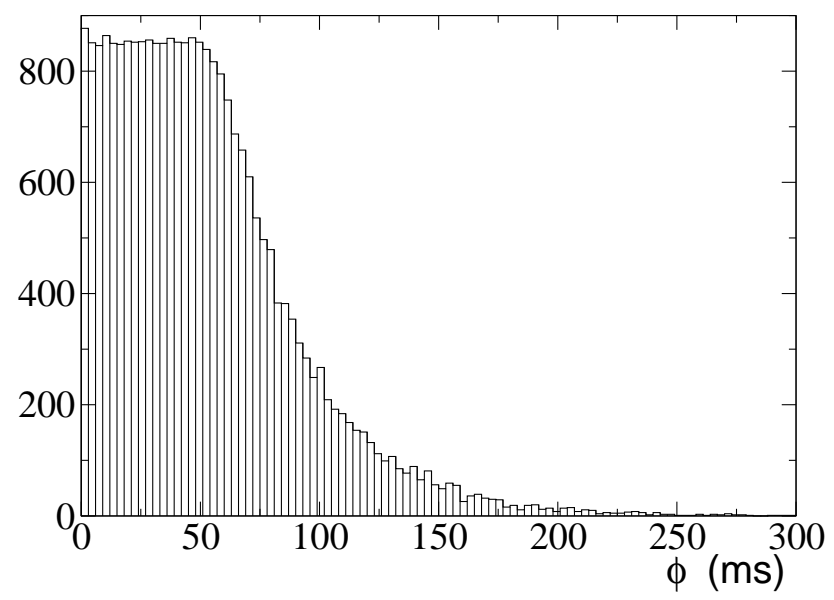

Figure 7: Histogram of the phases of the neurons.

The mean spike interval is obtained from the self-consistent equation

$$
\frac{<T>}{\tau}=\int_{0}^{\infty} d u \mathrm{e}^{-u^{2}}\left[\frac{\mathrm{e}^{2 y_{\theta} u}-\mathrm{e}^{2 y_{r} u}}{u}\right]
$$

with

$$
y_{\theta}=\frac{\theta-\mu}{\sigma}, \quad y_{r}=\frac{V_{r}-\mu}{\sigma}
$$

If $y_{\theta}$ is large, i.e. if the fluctuations of the potential are small compared to the difference between the driving potential $\mu$ and the threshold $\theta$, then the mean first passage time can be approximated by

$$
\frac{<T>}{\tau} \simeq \mathrm{e}^{\mathrm{y}_{\theta}^{2}}
$$

For the parameters of our simulations of the previous section, Eqs. (1113) give $\langle T\rangle=94.6 \mathrm{~ms}$ and $\langle T\rangle=93.5 \mathrm{~ms}$ which is in reasonable agreement with the mean spike interval of the simulations, $\langle T\rangle=97.6 \mathrm{~ms}$. The effective average stimulus $\mu$ is only $0.05 \mathrm{mV}$ below the threshold potential $\theta$, but this difference is still large compared to the fluctuations, one finds $y_{\theta}=1.23$.

Note that, contrary to the deterministic case, the mean potential relaxes to a value $\mu$ below the threshold $\theta$. The spikes are generated by fluctuations which cross the threshold. Nevertheless, in both cases, the value of $\mu$ is close to $\theta$ which almost gives an identical average spike interval, Eq.(6). In particular, the spike rate increases linearly with the stimulus $V_{\infty}$. 
It might be interesting to consider the limit of large networks, $N \rightarrow \infty$ with constant $J N=C$. Inserting the average spike interval, Eq.(6), into Eq.(13) yields

$$
\begin{aligned}
\left(\frac{\theta-\mu}{\sigma}\right)^{2} & =\ln \frac{p C}{V_{\infty}-\theta} \\
\sigma^{2} & =\frac{C}{N}\left(V_{\infty}-\theta\right) \\
(\theta-\mu)^{2} & =\frac{C}{N}\left(V_{\infty}-\theta\right) \ln \frac{p C}{V_{\infty}-\theta}
\end{aligned}
$$

Hence the fluctuations of the synaptic noise show an unusual behaviour in this limit: Its variance decreases with increasing system size $N$. However, the fluctuations relative to the difference between driving potential $\mu$ and threshold $\theta$ remain constant, yielding a constant mean spike interval $\langle T\rangle$. The fluctuations do not disappear in the thermodynamic limit $N \rightarrow \infty$.

\section{Discussion}

A homogeneous neural network with purely inhibitory synapses relaxes to a state of high attention. A large fraction of neurons have a membrane potential which is just below threshold. Hence the network can immediately - on the time scale of synaptic transmission - react on external excitatory pulses.

Although, at any time, the majority of the neurons accumulate closely below threshold, each single neuron fires irregularly with a low rate. In our model the broad distribution of spike intervals stems from synaptic unreliability; a synapse transmits the incoming spike with some probability, only. The numerical simulations show that the spiking events of the total network are correlated in time. Uncorrelated synaptic noise still leads to correlated spike intervals, even for infinite-range models.

For our model, in the thermodynamic limit any neuron receives an infinite number of synaptic inputs. Usually, one argues that, in this limit, the fluctuations of the synaptic input can be ignored. Our model, however, shows that even for an infinite number of inhibitory inputs the fluctuations of the membrane potential have a large effect. The network adjusts itself such that there remains a broad distribution of spike intervals in this limit.

The stationary state of the network is a pool of neurons firing irregularly with a low rate. The firing rate increases linearly with a global external stimulus. The network can immediately react to 
external pulses. Our model contains unreliable inhibitory synapses, only. Hence a network does not need to balance large excitatory and inhibitory synaptic currents to achieve these properties.

Our investigations concentrated on a very simple model: Integrateand-fire neurons with unreliable inhibitory synapses which couple all neurons. Each neuron has identical parameters. One may wonder whether and how the properties of the network change when the model is extended to other mechanisms. In fact, much is known about IFnetworks. For instance, synaptic delay leads to clusters of synchronized activity [7] and to oscillating firing rates [6]. A distribution of the number of short-range synaptic contacts increases the correlations between consecutive spike intervals [9]. Excitatory synapses can produce synfire chains, i.e. waves of synchronized activities moving through the network [1, 3]. When the excitation is too strong, the neurons synchronously fire at a high rate [5]. A distribution of threshold values obviously leads to a distribution of firing rates. In fact, I find that in this case, the stationary state of the network contains a fraction of neurons which never fire.

All these extensions modify the properties of state of irregularly firing neurons. However, one of the main questions remains unsolved: How does the network process information on top of this background activity?

\section{Acknowledgement}

The author would like to thank Moshe Abeles, Ramon Huerta and Ido Kanter for useful comments.

\section{References}

[1] Abeles M (1991) Corticonics, Cambridge University Press

[2] Allen C and Stevens CF (1994) An evaluation of causes for unreliability of synaptic transmission, Proc. Natl. Acad. Sci. USA 91: 10380-10383

[3] Aviel Y, Mehring, Abeles M and Horn D (2003) On embedding synfire chains in a balanced network, Neural Comp. 15(6): 13211340

[4] Aviel Y, Horn D, Abeles M (2005) Memory capacity of balanced networks, Neural Comp. 17: 691-713

[5] Brunel N (2000) Dynamics of sparsely connected networks of excitatory and inhibitory spiking neurons, J. Comp. Neuroscience 8: $183-208$ 
[6] Brunel N and Hakim V (1999) Fast global oscillations in networks of integrate-and-fire neurons with low firing rates, Neural Computation 11: 1621-1671

[7] Ernst U, Pawelzik K and Geisel T (1995) Synchronization induced by temporal delays in pulse-coupled oscillators, Phys. Rev. Lett. 74: $1570-1574$

[8] Gerstner W and Kistler W (2002) Spiking Neuron Models, Cambridge University Press

[9] Hertz JA, Richmond BJ and Nilsen K (2003) Anomalous response variability in a balanced cortical network, Neurocomp. 52-54: 787792

[10] Mirollo RE and Strogatz SH (1990) SIAM J. Appl. Math. 50: 1645

[11] Kestler J and Kinzel W (2006), Multifractal distribution of spike intervals for two neurons coupled by unreliable pulses, J. Phys A 39: L461-L466

[12] Lisman JE (1997) Bursts as a unit of neural information: making unreliable synapses reliable, Trends Neurosci. 20: 38-43

[13] Shadlen MN and Newsome WT (1994) Noise, neural codes and cortical organization, Curr. Opin. Neurobiol. 4(4): 569-579

[14] Van Vreeswijk,C., and Sompolinsky,H., Chaos in neuronal networks with balanced excitatory and inhibitory activity, Science 274, 1724-1726 (1996)

[15] Strogatz SH (2001), Nonlinear dynamics and chaos, Cambridge University press

[16] Timme M, Wolf F and Geisel T (2002) Coexistence of regular and irregular dynamics in complex networks of pulse-coupled oscillators, Phys. Rev. Lett. 89: 258701

[17] Trevelyan AJ and Watkinson O (2005) Does inhibition balance excitation in neocortex? Progress in Biophysics and Molecular Biology 87 109-143

[18] Tuckwell HC (1998) Introduction to theoretical neurobiology, Cambridge University Press

[19] Zillmer R, Livi R, Politi A and Torcini A (2006) Desnychronized stable states in diluted neural networks, cond-mat/0608188 